\title{
p63 null mutation protects mouse oocytes from radio-induced apoptosis
}

\author{
Gabriel Livera ${ }^{1,2,3}$, Béatrice Petre-Lazar ${ }^{1,2,3}$, Marie-Justine Guerquin ${ }^{1,2,3}$, Emilie Trautmann ${ }^{1,2,3}$, \\ Hervé Coffigny ${ }^{1,2,3}$ and René Habert ${ }^{1,2,3}$ \\ ${ }^{1} C E A, D S V / D R R / S E G G / L D R G$, Unit of Gametogenesis and Genotoxicity, Laboratory of Differentiation and \\ Radiobiology of the Gonads, F-92265 Fontenay aux Roses, France, ${ }^{2}$ Univ Paris 7-Denis Diderot, UFR of Biology, \\ UMR-S 566, F-92265 Fontenay aux Roses, France and ${ }^{3}$ INSERM, U566, F-92265 Fontenay aux Roses, France \\ Correspondence should be addressed to G Livera at Unit of Gametogenesis and Genotoxicity, CEA, Université Paris 7, INSERM \\ U566, CEA/DSV/DRR/SEGG/LDRG, Route du Panorama-BP6, 92265 Fontenay aux Roses Cedex, France; \\ Email: gabriel.livera@cea.fr
}

\begin{abstract}
Female fertility in mammals is determined by the pool of primordial follicles and low doses of radiation induce a major loss of primordial follicles in the ovary. We investigated the expression of p53 and its homologues, p63 and p73, in the normal and irradiated neonatal ovary. p63 was the only member of the p53 family detected in oocyte nucleus. No p63 transcripts or protein were detected in the early foetal ovary. p63 production began in late pachytene-stage oocytes and peaked in diplotene oocytes in mice and humans. The production of p63 was correlated with meiotic DNA double-strand break repair. Only transactivation (TA) isoforms were present in the ovary, with TAp63 $\alpha$ by far the most abundant in terms of mRNA and protein levels. Complete p63 null mutation did not affect normal ovary development. Irradiation rapidly triggered p63 phosphorylation. p63 null mutation prevented the cleavage of caspases-9 and -3 and the follicle loss induced by ionising radiation. Thus, our results evidence that irradiation-induced depletion of the primordial follicle pool results from the activation of p63 in quiescent oocytes.

Reproduction (2008) 135 3-12
\end{abstract}

\section{Introduction}

Female fertility depends on the size of the primordial follicle pool. Primordial follicle formation is initiated late in foetal development and is completed at or shortly after birth (Skinner 2005). The major side effects of chemotherapy and radiotherapy frequently include ovarian failure and infertility (Meirow \& Nugent 2001). Oocytes from primordial follicles are extremely sensitive to radiation, with the pool of primordial follicles in young adult female mouse ovaries almost completely destroyed within 2 weeks of a single exposure to 0.1 Gy of ionising radiation (IR; Morita et al. 2000). We recently reported that caspase-2 plays a key role in the radiation-induced death of oocytes (Hanoux et al. 2006). However, caspase-2 alone cannot prevent oocyte apoptosis, and other pathways must therefore be activated during this process.

Radiation exposure induces cell death via apoptosis a regulated process allowing the elimination of damaged cells. The major effects of IR on cells include the production of DNA double-strand breaks (DSBs), triggering the mitochondrial apoptotic pathway (Prise et al. 2005). Germ cells have long been known to be very sensitive to genotoxic stress and DNA damage. We recently reported that this sensitivity changes considerably during ovarian development. Indeed, mouse oocytes in the early stages of prophase I of meiosis (leptotene, zygotene and pachytene) are more resistant to high doses of IR than their oogonial precursors or later diplotene/diakinesis-stage oocytes (Hanoux et al. 2006). Foci of phosphorylated histone $\mathrm{H}_{2} \mathrm{AX}(\gamma \mathrm{H} 2 \mathrm{AX})$ have been observed in irradiated diplotene oocytes. These foci appear shortly before the sequential activation of caspases-9 and -3 (Hanoux et al. 2006).

The p53, p63 and p73 proteins form a family of transcription factors involved in cellular responses to stress and development. These three genes encode multiple p53, p63 and p73 proteins containing different protein domains, due to multiple splicing, and the use of alternative promoters and translation initiation sites (Levrero et al. 2000). The p63 and p73 proteins have been classified into two groups on the basis of their $\mathrm{N}$-termini, TA and $\Delta \mathrm{N}$. The $\mathrm{p} 63$ gene encodes only six isoforms (Yang et al. 1998), whereas p73 has a more complex distribution, with additional $\mathrm{N}$ - and C-terminal splice variants (Melino et al. 2002). The full-length TA isoforms have a transactivation domain and function 
similarly to $\mathrm{p} 53$. By contrast, $\Delta \mathrm{N}$ isoforms are truncated; they lack this domain and function in an opposite manner to TA isoforms. Alternative splicing also occurs at the $\mathrm{C}$-terminus, generating three different transcripts for each group (TA and $\Delta N$ ): $\alpha$ for the entire RNA, $\beta$ and $\gamma$ for the shortest RNA. The $\alpha$ isoforms contain the sterile alpha motif (SAM) domain involved in protein-protein interactions. This motif has been implicated in various processes, including development, differentiation, apoptosis, focal adhesion and chromatin remodelling, and is absent from p53 (Westfall \& Pietenpol 2004).

Recently, the specific null mutation of TAp63 has been reported to prevent oocyte apoptosis induced by IR (Suh et al. 2006). In the current report, we used null mutants for all p63 isoforms. Using this model, we confirmed and extended the function of p63 in the DNA damage-induced apoptosis of primordial follicles. We also investigated the expression of p53, p63 and p73 in the neonatal mouse ovary, the stage identified as the most sensitive to genotoxic stress. Our data demonstrated that TAp63 $\alpha$ is the main member of the p53 family expressed in the nucleus of oocytes after meiotic DSB repair.

\section{Results \\ p63 is expressed in the oocyte nucleus}

We localised p53, p63 and p73 by immunohistochemistry in sections from 8 days postpartum (dpp) ovaries. No specific signal for p53 was obtained in small oocytes (Fig. 1A). Large growing oocytes
A 8 dpp ovary
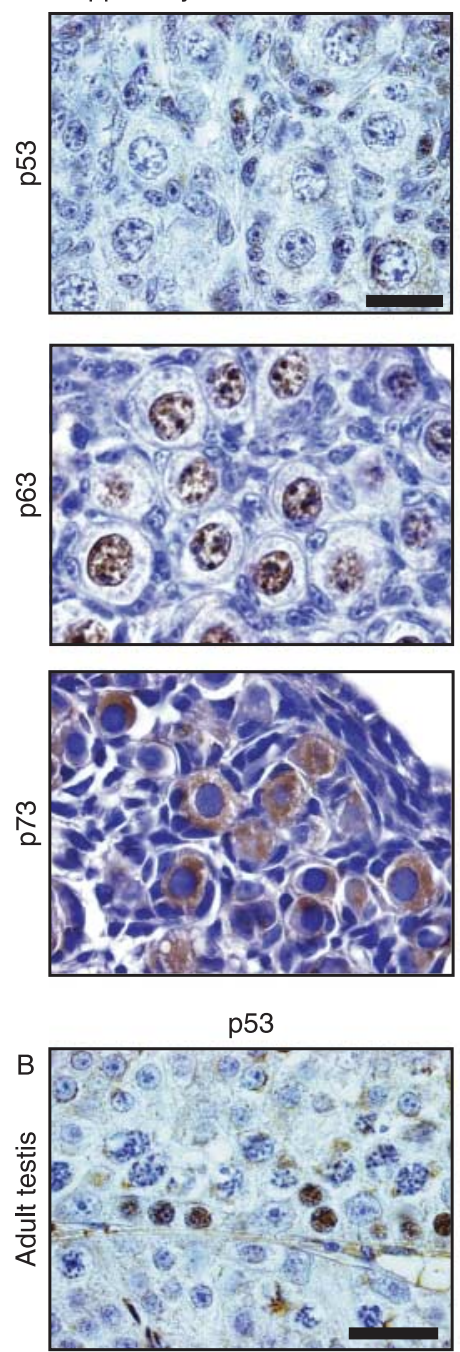
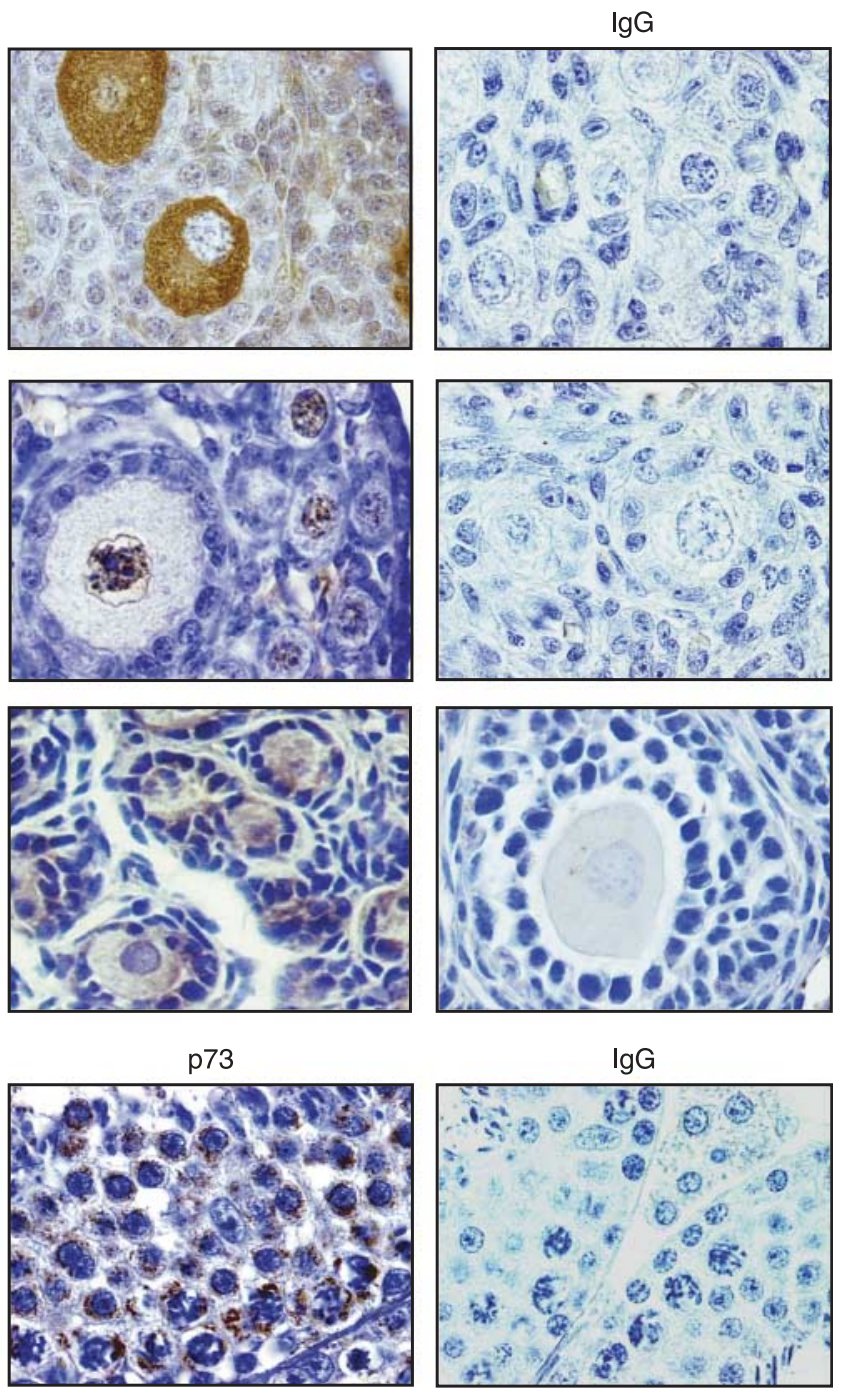

Figure 1 p53 and homologues in the neonatal ovary. (A) Immunohistochemical staining of p53, p63 and p73 was carried out on sections of 8 dpp wild-type ovaries containing small quiescent follicles (left panels) and growing follicles (middle panels). (B) Sections of adult wild-type testes were used as a positive control for p53 and p73 immunohistochemical staining. Negative controls in which the primary antibody has been replaced by non-immune IgG are presented (right panels). Images presented are representative of at least four different experiments. The scale bar corresponds to $20 \mu \mathrm{m}$. 
displayed strong cytoplasmic labelling. Testes from adult wild-type mice were used as a positive control as p53 expression has previously been reported in spermatogonia (Beumer et al. 1998). We obtained a similar pattern of staining (Fig. 1B). We localised p63 using the 4A4 antibody that specifically recognises all isoforms of p63. We detected large amounts of p63 protein specifically in the nucleus of small resting and growing oocytes (Fig. 1A). We detected p73 only in the cytoplasm of the oocytes. As previously described (Hamer et al. 2001), p73 was also found in the cytoplasm of adult testicular germ cells (Fig. 1B). We then determined the distributions of these three p53 family members in the ovary 2 and $4 \mathrm{~h}$ after irradiation $(0.5 \mathrm{~Gy})$. We found that $\gamma$-radiation had no effect on the expression of p53, p63 and p73 (data not shown). When corresponding non-immune $\operatorname{lgG}$ was used in place of antibodies to p53, p63 or p73, no staining was observed (Fig. 1, right panels). The specificity of the staining for p63 was further confirmed using section of p63 null ovaries which gave no signal (see below).

\section{p63 expression is initiated in late prophase I in mouse and human oocytes}

We investigated p63 expression in more detail, as a function of the developmental stage of the ovary. Immunohistochemical staining for p63 gave no specific signal in early mouse foetal ovaries, from 12.5 to 16.5 days postconception (dpc), containing oogonia and leptotene-, zygotene- and early pachytene-stage oocytes (Fig. 2A). In $18.5 \mathrm{dpc}$ ovaries, a weak signal was detected in about onethird of the oocytes. No correlation was observed between oocyte meiotic stages and staining, as both late pachytene and diplotene oocytes were labelled at this age. The signal increased in intensity shortly after birth, with almost all oocytes reaching the diplotene stage and being labelled. We carried out similar observations with sections from human ovaries. No staining was observed in the oogonia and during early prophase I, using ovaries obtained at between 7 and 15 weeks of gestation (Fig. 2B). Strong staining of late pachytene- and diplotene-stage oocytes was detected in ovaries obtained at 24 and 26 weeks of gestation.

\section{The production of p63 is correlated with DNA DSB repair}

DSBs in DNA are induced by genotoxic stresses, such as IR, but are also produced during the early steps of prophase I of meiosis. We used immunofluorescence techniques to search for $\gamma-\mathrm{H} 2 \mathrm{AX}$ foci, a marker of DSBs (Fig. 3). Using $\gamma-\mathrm{H} 2 \mathrm{AX} / \mathrm{p} 63$ double staining in ovaries obtained from $17.5 \mathrm{dpc}$ to $3 \mathrm{dpp}$, we detected very little double labelling of oocytes (Fig. 3A-D). Most of the oocytes presented $\gamma-\mathrm{H} 2 \mathrm{AX}$ staining at $17.5 \mathrm{dpc}$, whereas very few were stained for p63. By
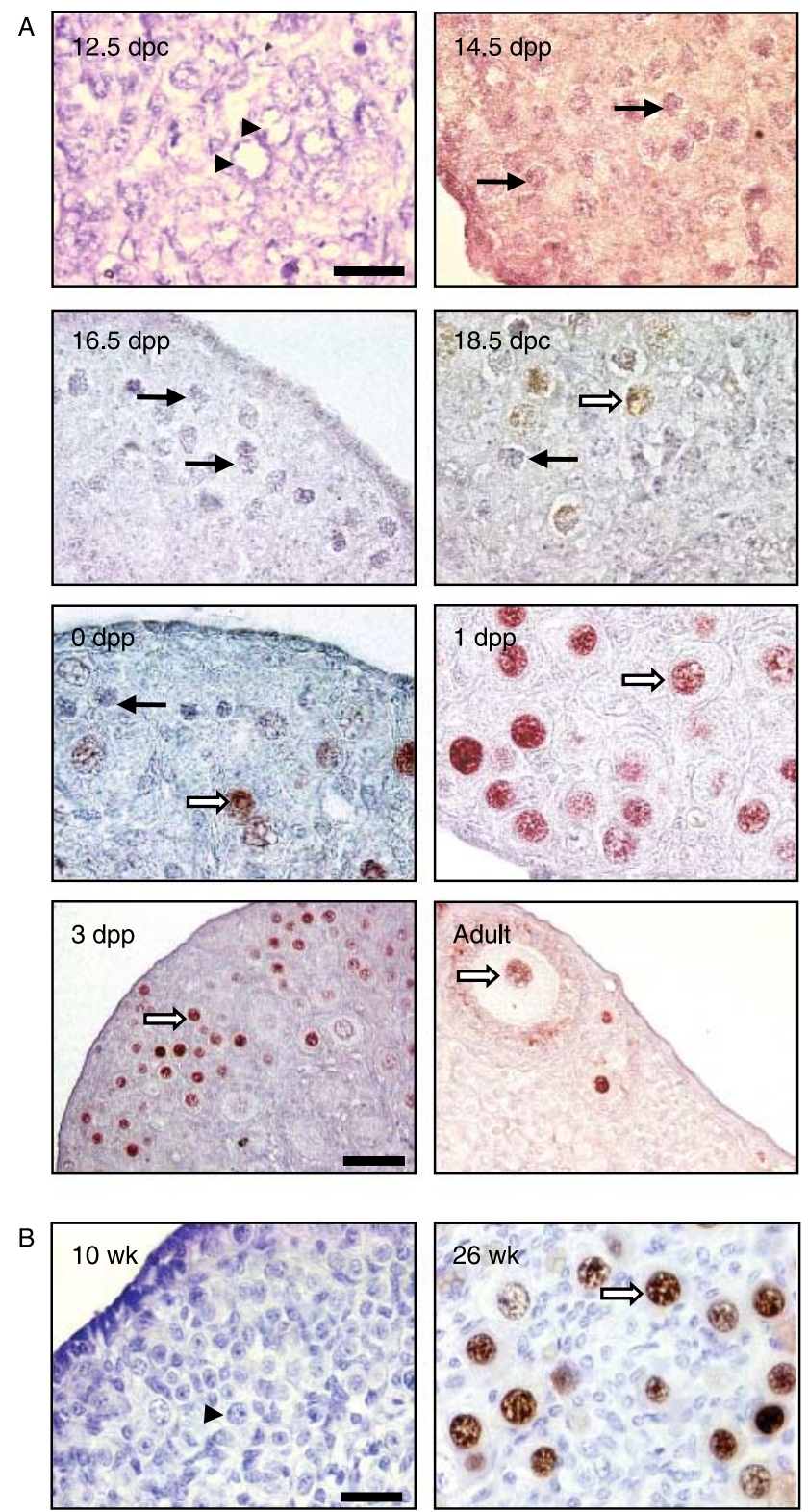

Figure 2 Distribution of p63 in the developing mouse and human ovary. (A) Immunohistochemical staining for p63 was performed with antipan-p63 antibody (4A4), on sections from foetal and neonatal mouse ovaries. (B) The same immunohistochemical procedure was carried out on sections of human foetal ovaries. Arrowheads indicate oogonia, fine arrows indicate unlabelled oocytes and white arrows indicate stained oocytes. Images presented are representative of three independent experiments. The scale bar corresponds to $20 \mu \mathrm{m}$, except in mouse 3 dpp and adult ovary sections, in which it corresponds to $50 \mu \mathrm{m}$.

contrast, from 18.5 dpc onwards, only a few oocytes were stained for $\gamma-\mathrm{H} 2 \mathrm{AX}$, and almost none of these oocytes displayed staining for p63, although many of the surrounding oocytes had clearly started to produce this protein. Similarly, in the adult testis, germ cells showed almost no co-localisation of $\gamma-\mathrm{H} 2 \mathrm{AX}$ and p63 (Fig. 3E and F). 


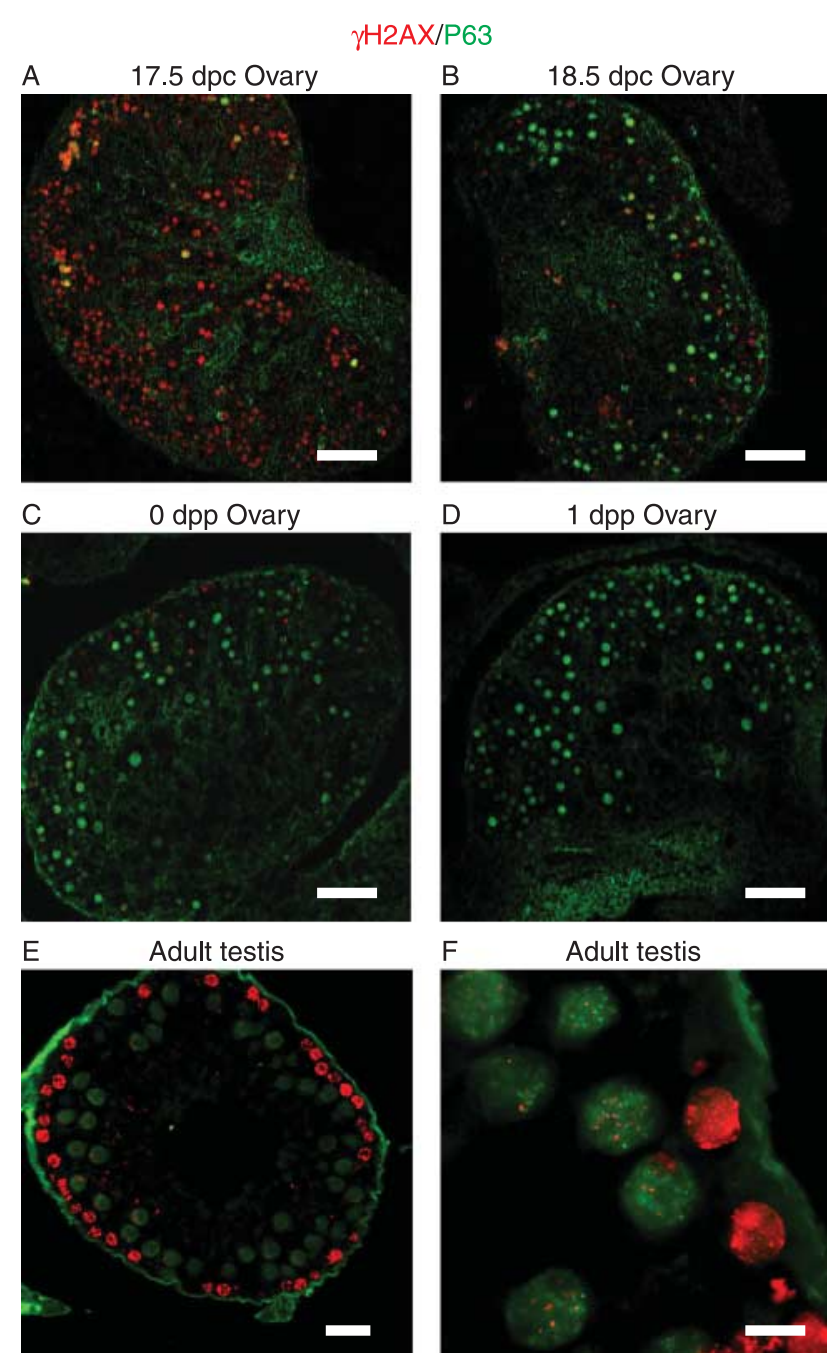

Figure 3 Absence of co-localisation of spontaneous meiotic DNA DSBs and p63. DSBs are labelled with $\gamma \mathrm{H} 2 \mathrm{AX}$ (red) and p63 (green) in sections of late foetal and early post-natal mouse ovaries (A-D; bar, $40 \mu \mathrm{m}$ ) and in sections of adult testis (E and F; bar, $10 \mu \mathrm{m}$ ). Images are representative of three independent experiments.

\section{TAp63 $\alpha$ is the main p63 isoform expressed in oocytes from primordial follicles}

We investigated levels of the various p63 mRNAs in foetal and neonatal ovaries using the PCR strategy described in Fig. 4A. Primers targeting a sequence common to all p63 isoforms generated no signal between 12.5 and $14.5 \mathrm{dpc}$ and a strong band from $18.5 \mathrm{dpc}$ onwards (Fig. 4B). A weak signal was detected on $16.5 \mathrm{dpc}$ when performing several additional PCR cycles. A similar pattern was obtained with primers specific for TA-isoforms, whereas no signal was observed for $\Delta \mathrm{N}$-isoforms. In $1 \mathrm{dpp}$ neonatal ovaries, RT-PCR generated a strong signal for $\alpha$-isoforms and a much weaker signal for $\gamma$-isoforms. No signal was detected for $\beta$-isoforms in the ovary.

Western blot analysis of p63 in 1 dpp ovaries confirmed high levels of TAp63 $\alpha$ production (Fig. 4C).

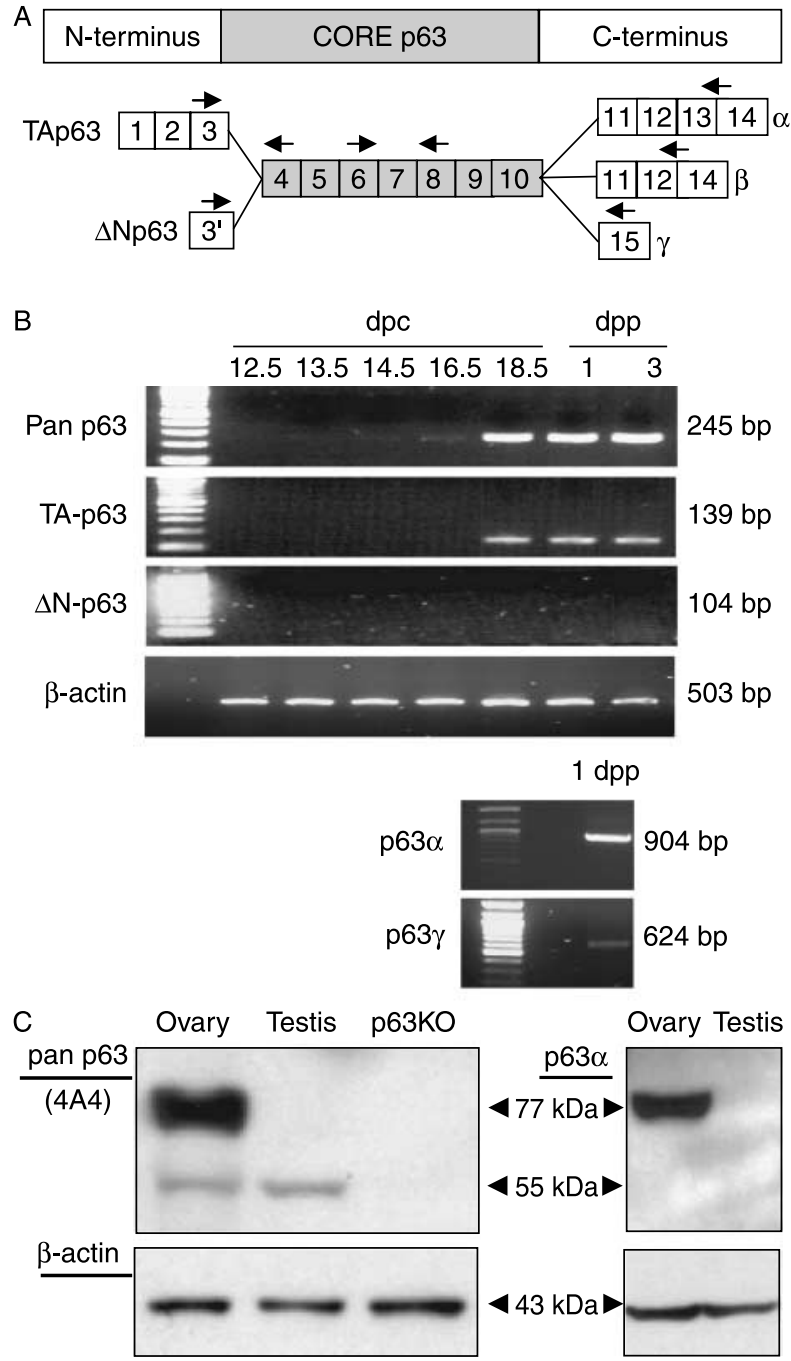

Figure 4 p63 mRNA and protein levels in the developing mouse ovary. The p63 family contains six isoforms. The PCR strategy used to amplify specific isoforms is shown in (A). RT-PCR was carried out for foetal and neonatal ovaries from $12.5 \mathrm{dpc}$ to $3 \mathrm{dpp}$ (B). The size of the amplicon obtained is given on the right. RT-PCR was repeated four times with pool of 8-20 ovaries. (C) Western blot analyses were performed on 1 dpp wild-type mouse ovaries and 18.5 wild-type and p63 null testes, using an antibody recognising all p63 isoforms (4A4; left panel) or an antibody specific to $p 63 \alpha$ (right panel). Molecular weights are given in the middle. Blots presented are representative of three to six experiments.

A much weaker signal corresponding to a $55 \mathrm{kDa}$ band being possibly TAp63 $\gamma$ was also detected after prolonged exposure. Western blots realised with the antibody specific to $p 63 \alpha$ showed only the band matching with TAp63 $\alpha$ molecular mass (i.e. $77 \mathrm{kDa}$ ) and not the $55 \mathrm{kDa}$ band. Immunohistochemical staining with a p63 $\alpha$-specific antibody also produced a strong nuclear signal, similar to that obtained with the pan p63 antibody, in mouse neonatal ovaries and human ovaries at 26 weeks of gestation (data not shown). p63 $\gamma$ could not be detected by immunohistochemistry. 


\section{p63 null mutation does not affect spontaneous ovary development}

We investigated the function of p63 using p63 null animals. As p63 null mice die within a few hours of birth (Mills et al. 1999, Yang et al. 1999), we removed the ovaries at 18.5 dpc (i.e. 1 day before birth) and cultured them for 7 days (equivalent to $6 \mathrm{dpp}$ ) to investigate the effect of p63 inactivation during the early folliculogenesis that normally occurs just after birth. At $18.5 \mathrm{dpc}$, wild-type and p63 null ovaries were grossly similar, with many oocytes in the late pachytene and diplotene stages. On day 7 (D7), wild-type ovaries contained numerous small follicles and a few growing follicles (Fig. 5). The invalidation of p63 had no obvious effect on the pattern of follicle formation.

We confirmed that p63 invalidation was effective in the ovary, by analysing p $63 \alpha$ levels by immunohistochemistry in wild-type and p63 null ovaries, at $18.5 \mathrm{dpc}$ and after culture (Fig. 5). We detected no p63 $\alpha$ in p63 - /- ovaries, whereas wild-type ovaries gave a moderate signal on 18.5 dpc and a much stronger signal after 7 days of culture. We then investigated whether culture or p63 null mutation modified the expression of other p53 family members. We carried out immunohistochemical staining of p53 and p73 in sections from p63 null and wild-type cultured ovaries. As in vivo (Fig. 1), in cultured wild-type ovaries, p53 and p73 were undetectable in the nucleus of the oocytes with p73 being consistently detected in the cytoplasm of the both small and growing oocytes and p53 being detected only in the cytoplasm of growing oocytes (data not shown). This pattern of staining was not modified by p63 null mutation.
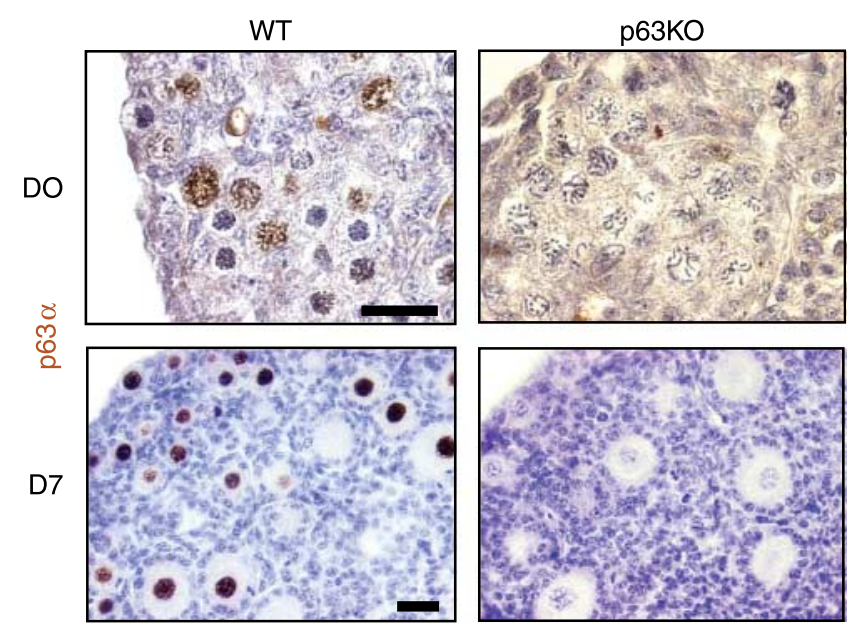

Figure 5 Effect of p63 invalidation on the developing ovary. As p63KO mice die at birth, p63 WT and KO ovaries were explanted $18.5 \mathrm{dpc}$ (D0), cultured for 7 days (D7), fixed and processed for histology. Immunohistochemical staining was carried out on sections from ovaries of both genotypes, using an antibody specifically recognising p63 $\alpha$ isoforms. Images given are representative of three experiments using ovaries from different animals.

\section{p63 null mutation prevents radiation-induced oocyte apoptosis}

We investigated the response of wild-type and p63 null ovaries to IR. We subjected $18.5 \mathrm{dpc}$ ovaries to irradiation with $3 \mathrm{~Gy}$, which we previously determined to be the lowest dose eradicating most oocytes at this stage, and cultured them for 7 days. At the end of culture, most of the oocytes from both p63 wild-type and p63 null animals died (Fig. 6A).

As p63 expression peaks a few days after $18.5 \mathrm{dpc}$, we cultured 18.5 dpc ovaries for 2 days and then subjected the explants to IR. At the end of the 2-day culture period, most of the oocytes were enclosed in primordial follicles (data not shown). Oocyte sensitivity to radiation had increased by this stage, as the lowest dose eradicating most of the wild-type oocytes was $0.5 \mathrm{~Gy}$. Most of the p63 null oocytes exposed to 0.5 Gy after 2 days of culture survived until day 7 of culture. At this point, we also detected cells expressing heat shock protein 90 (HSP90) a germ cell marker (Fig. 6B). Many HSP90positive cells were detected in irradiated p63 null ovaries, whereas few were present in the irradiated wild-type ovary. Interestingly, most of the few surviving wild-type oocytes present in irradiated ovaries at the end of culture were enclosed in growing follicles (Fig. 6C), whereas most of the many p63 null oocytes were present in small primordial follicles. Finally, we determined the percentage of oocytes stained for cleaved caspases- 3 and $-9,6 \mathrm{~h}$ after irradiation. In wild-type ovaries, irradiation with 0.5 Gy on day 2 induced a massive increase in the number of oocytes labelled for cleaved caspases-3 and 9 (Fig. 6D and E). This increase was completely abolished in p63 null mutants.

In several experiments, p63 null ovaries were exposed to a 3 Gy dose after day 2 of culture. In these ovaries, hardly any oocytes were retrieved on day 7 of culture (data not shown).

\section{Irradiation induces 063 phosphorylation}

We investigated whether p63 was activated in response to IR. We assessed $\mathrm{p} 63$ protein levels by Western blotting shortly after the irradiation of $1 \mathrm{dpp}$ ovaries with $0.5 \mathrm{~Gy}$. As early as $1 \mathrm{~h}$ post-irradiation, we observed a shift of the TAp63 $\alpha$ band (Fig. 7A). The signal for the shifted band was weak at $1 \mathrm{~h}$ and the shift was almost complete $6 \mathrm{~h}$ post-irradiation. Treatment of whole protein extracts of irradiated ovaries with alkaline phosphatase for $20 \mathrm{~min}$ reversed this change in the molecular weight of p63, confirming that irradiation induced the phosphorylation of p63 in the ovary.

In neonatal testes submitted to a 0.5 Gy dose of $\gamma$-rays, only the $55 \mathrm{kDa}$ band matching with p63 $\gamma$ molecular weight was retrieved. Irradiation induced no obvious shift of this band. 

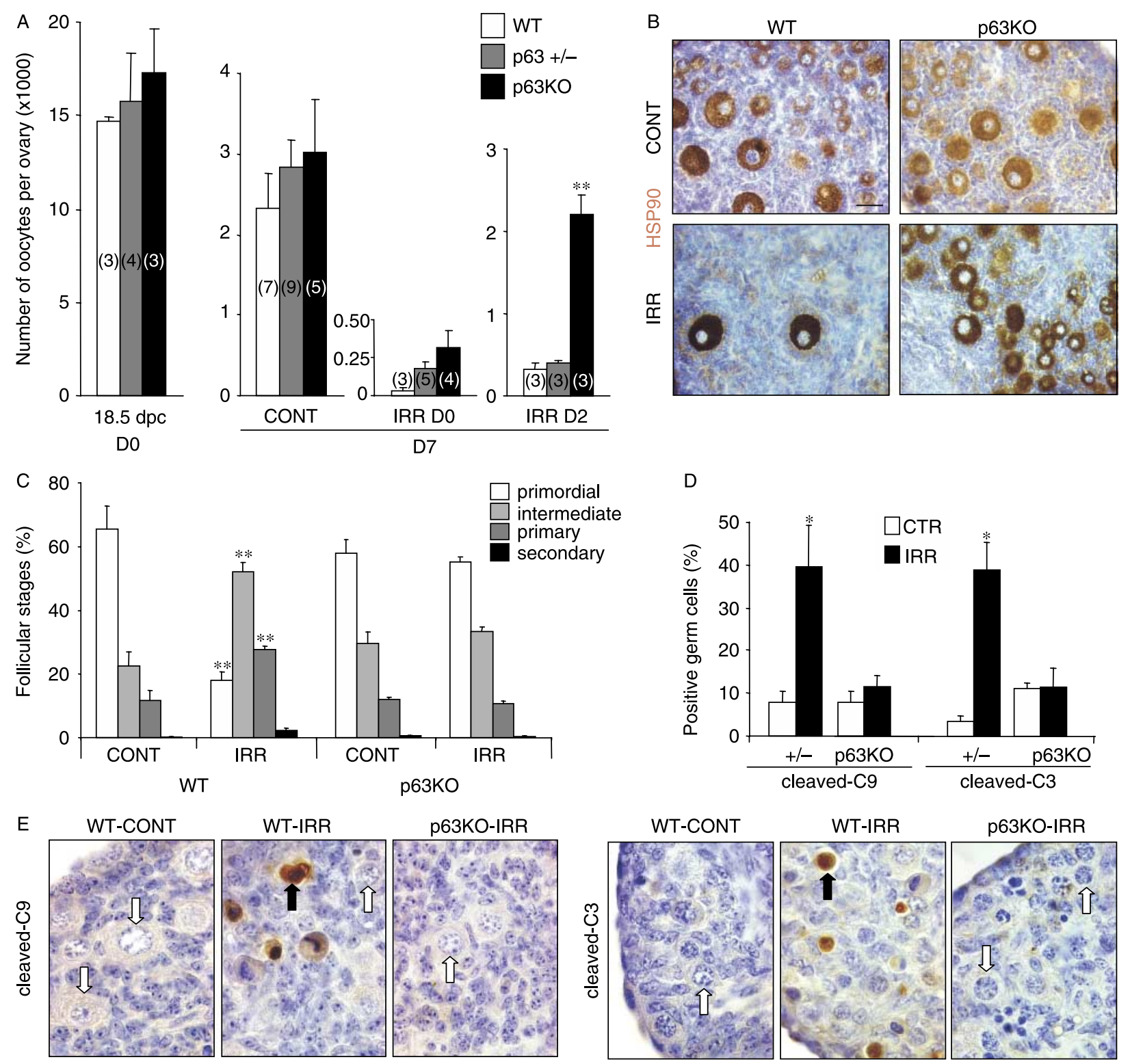

Figure 6 Effect of p63 null mutation on the number of oocytes. (A) The total number of germ cells per ovary was determined in p63 wild-type (WT), heterozygous (+/ ) and homozygous null (KO) ovaries from $18.5 \mathrm{dpc}$ foetuses (D0) and after 7 days in organ culture (D7). Some ovaries were irradiated with 3 Gy on day 0 (IRR D0) or with 0.5 Gy on day 2 (IRR D2) and others were not irradiated (CONT). Columns show the means \pm s.E.M. of determinations. The number of ovaries is given in brackets. (B) Immunostaining of HSP90, a marker of germ cells, was carried out in sections of p63 WT and KO sham-irradiated ovaries (CONT) and in ovaries irradiated with 0.5 Gy on day 2 (IRR). (C) The distribution of the follicular stages present on day 7 was determined in the same ovaries as in B. (D and E) The percentages of cleaved caspase-9-positive (cleaved-C9) and cleaved caspase-3positive germ cells (cleaved-C3) were determined in ovaries treated as in B and fixed $6 \mathrm{~h}$ after irradiation. The white arrows indicate unstained oocytes and the black arrows indicate caspase-positive cells. $* P<0.05$ and $* * P<0.01$ for statistical comparisons between $p 63+/+$ and $p 63+/-$ or $-/-$, using Student's $t$-test.

\section{Discussion}

We show here that TAp63 $\alpha$ is expressed in the oocyte and activated in response to IR, triggering apoptotic pathways leading to follicular depletion.

Our results are entirely consistent with the data recently published by McKeon's group (Suh et al. 2006). Indeed, this group evidenced that p63 was phosphorylated in response to IR and demonstrated that this phosphorylation corresponded to an increase in the transcriptional activity of p63. We also retrieved a similar phosphorylation pattern and found that downstream effectors, such as caspases- 3 and -9 , were not activated in the absence of p63. TA-p63 isoforms, as p53, can activate several apoptosis pathways, while $\Delta \mathrm{N}-\mathrm{p} 63$ isoforms can inhibit both TA-p63 and p53 activities. 

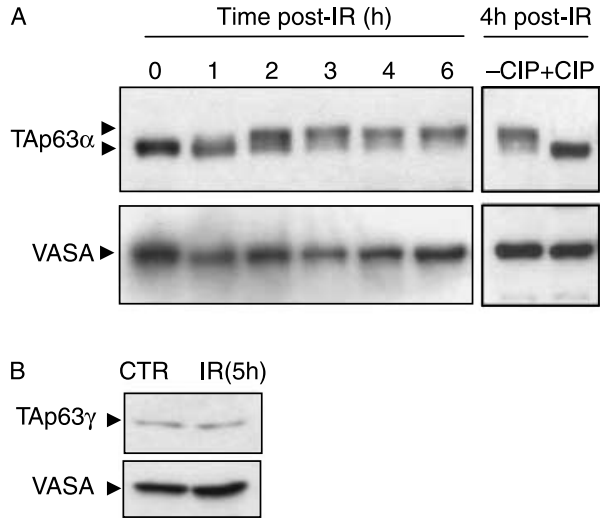

Figure 7 Detection of p63 phosphorylation following exposure to ionising radiation. p63 (4A4 antibody) and VASA $(\mathrm{MVH})$ were detected by Western blotting in $1 \mathrm{dpp}$ ovaries (A) and testes (B) before and at various times after $0.5 \mathrm{~Gy}$ irradiation. Some lysates of irradiated ovaries were treated with CIP phosphatase $(+\mathrm{CIP})$ to assess p63 phosphorylation. Images represent three to five independent experiments using pools of 10-20 ovaries and three or four testes.

McKeon's group showed that the specific null mutation of TA-p63 prevented the loss of the follicular reserve in response to radiation. We report here that oocytes from null mutants for all p63 isoforms are similarly protected. These data rule out the possibility that $\Delta \mathrm{N}$-p63 isoforms protect female germ cells. Overall, these data indicate that $\mathrm{p} 63$ plays a unique and upstream role in the commitment of the oocyte to the cell death pathway following genotoxic stress. Several other factors, such as sphingosine 1-phosphate and caspase2 , have been shown to regulate or to be involved in this stress-induced death, but none has been shown both to abolish oocyte apoptosis entirely and to be activated in response to genotoxic stress (Morita et al. 2000, Hanoux et al. 2006).

We also provide evidence that p63 is expressed in mouse and human oocytes at the end of prophase I, mostly at the diplotene stage. We recently reported considerable changes in oocyte radiosensitivity during prophase I, with the zygotene stage being resistant to high doses of IR, the pachytene stage also showing some degree of radioresistance and the diplotene stage being highly radiosensitive (Hanoux et al. 2006). The expression of p63 therefore seems to be correlated with oocyte radiosensitivity to genotoxic stress. One of the main features of the damage caused by genotoxic stress is DNA DSBs. In this regard, our current report that p63 is not normally expressed in oocytes containing meiotic DNA DSBs is particularly interesting. IR rapidly induces new DNA DSBs in diplotene oocytes (Hanoux et al. 2006, Suh et al. 2006). These DSBs may be detected by a kinase that then phosphorylates p63, activating the transcription of pro-apoptotic factors. However, the kinase involved and the sites of p63 phosphorylation remain to be identified.
Apoptosis can still be induced in primordial oocytes from p63 null animals when these are submitted to higher doses of radiation. This suggests that p63-independent apoptotic pathways must exist. We reported here that p53 was absent from the oocyte nucleus. These data fit with the previously proposed hypothesis that p53 is not involved in the DNA damage-induced death of primordial oocytes (Suh et al. 2006). P73 was also absent of the oocyte nucleus but was detected in the cytoplasm of the primordial oocyte. In the testicular germ cells, cytoplasmic p73 has been detected and was proposed to represent an apoptotic pathway activated in response to high doses of radiation (Hamer et al. 2001). The same may be true in the ovary with the p63 route being the primary apoptotic pathway and p73 being a less sensitive pathway. The hetero-oligomerisation of p63 and p73 has been observed (Davison et al. 1999, Gaiddon et al. 2001). In the present study, we identified p63 as the only member of the p53 family present in the nuclei of diplotene oocytes. This suggests that p63 cannot directly interact with p73 in the oocyte and that both pathways are independent.

The main p63 isoform identified in the diplotene oocyte was TAp63 $\alpha$. The transfection of a cell line with a construct encoding TAp63 $\alpha$ has been shown to induce the mitochondrial apoptotic pathway in response to chemotherapeutic drugs (Gressner et al. 2005). We reported a similar p63-dependent induction of the mitochondrial apoptotic pathway in the oocytes with cleavage of caspase-9. Interestingly, p63 $\alpha$ isoforms are the only isoforms of this protein containing the C-terminal SAM domain, which inhibits the p63 activation domain, presumably through intramolecular interactions (Serber et al. 2002). Our results suggest that p63 phosphorylation probably prevents the inhibitory effects of this C-terminal domain.

The difference in basal levels of TAp63 $\alpha$ in mitotic and meiosis-arrested female germ cells seems to be correlated with the radiosensitivity of these cells. Indeed, in mice, oogonia undergo high levels of apoptosis only if exposed to more than $1.5 \mathrm{~Gy}$, whereas diplotene oocytes are much more radiosensitive, with apoptosis occurring even at doses of only 0.1 Gy. The high level of TAP63 $\alpha$ isoform production in differentiated oocytes probably predisposed these cells to respond to genotoxic stress by committing suicide. Thus, p63 $\alpha$ seems to be involved in detecting DNA damage in germ cells specifically after prophase I of meiosis, when the repair of meiotic DNA DSBs has been completed. This is consistent with the recent proposal that p63 acts as the guardian of the germ cell genome, but narrows this role specifically to female differentiated germ cells (i.e. post-prophase I). In this regard, our previous report that almost only TAp63 $\gamma$ protein was detectable in mitotic male germ cells and that spontaneous germ cell apoptosis was decreased in p63 null testes is particularly interesting (Petre-Lazar et al. 2007). One may then hypothesise that TAp63 isoforms play a fundamental and 
bivalent role in promoting germ cell apoptosis with TAp63 $\gamma$ being present in male germ cells and being constitutively active, while TAp63 $\alpha$ is only expressed in female germ cells and is activated only in response to DNA damage.

In conclusion, we report here essential data concerning the involvement of p63 in DNA damageinduced depletion of the primordial follicle stock. We demonstrate that TAP63 $\alpha$ is phosphorylated in response to genotoxic stress, leading to the induction of apoptosis in quiescent oocytes. We found that p63 expression was correlated with changes in oocyte radiosensitivity and the disappearance of meiotic DNA DSBs. TAp63 $\alpha$ is thus a promising target for future studies aiming to preserve ovarian function after radioor chemotherapy.

\section{Materials and Methods}

\section{Animals, whole-body $\gamma$-irradiation and tissue processing}

Female NMRI mice were housed under controlled photoperiod conditions (lights on 0800-2000 h) and were supplied with commercial feed and tap water ad libitum. The day after an overnight mating was counted as day 0.5 post-conception (0.5 dpc). Natural birth occurred on $19.5 \mathrm{dpc}$, which was counted as day 0 postpartum ( $0 \mathrm{dpp})$. All animal studies were conducted in accordance with the NIH Guide for the Care and Use of Laboratory Animals. Ovaries were collected from $12.5 \mathrm{dpc}$ to $8 \mathrm{dpp}$. They were fixed immediately in Bouin's fluid or in $4 \%$ paraformaldehyde for at least $1 \mathrm{~h}$, dehydrated in alcohol, embedded in paraffin and cut into $5 \mu \mathrm{m}$ sections.

IR-induced follicular death was studied in neonatal ovaries. Animals were exposed to whole-body $\gamma$-irradiation at 1 and 8 $\mathrm{dpp}$, using a ${ }^{137} \mathrm{Ce}$ isotopic source. They were exposed to a total dose of $0.5 \mathrm{~Gy}$, administered at a rate of $0.62 \mathrm{~Gy} / \mathrm{min}$. Ovaries were collected $0,1,2,3,4$ and $6 \mathrm{~h}$ after irradiation. Ovaries were processed for immunohistochemistry as described above. For Western blotting, ovaries were frozen in liquid nitrogen and stored at $-80^{\circ} \mathrm{C}$ until protein extraction. Adult and developing testes were also dissected out and fixed or frozen similarly.

P63-/- embryos were produced by intercrossing heterozygous P63Brdm2 (+/-) mice (C57BL/6 genetic background) purchased from Jackson Laboratories (Bar Harbor, ME, USA). The targeted disruption of the murine p63 gene has been described elsewhere (Mills et al. 1999). At 18.5 dpc, p63-/mice could be identified visually based on their limbless phenotype. Embryos were also genotyped by PCR using the following primers to amplify the mutant p63 allele: F $5^{\prime}$ GTGTTGGCAAGGATTCTGAGACC $3^{\prime}$ and R $5^{\prime}$ GGAAGACAATAGCAGGCATGCTG $3^{\prime}$.

\section{Organ culture}

Ovaries were collected at $18.5 \mathrm{dpc}$, cut open and placed on a Millicell filter (pore size: $0.45 \mu \mathrm{m}$; Millipore, St Quentin en Yvelines, France). The filter was floated on Dulbecco's modified
Eagle's medium: Ham's-F12 (50\% v/v; Invitrogen) supplemented with $15 \mathrm{mM}$ HEPES (Invitrogen), $40 \mu \mathrm{g} / \mathrm{ml}$ gentamycin (Sigma), $1 \%$ foetal calf serum (FCS; Invitrogen) in 24-well culture plates (Nunc) and incubated at $37^{\circ} \mathrm{C}$, under an atmosphere containing $5 \% \mathrm{CO}_{2}$. After 2 or $48 \mathrm{~h}$ of culture, organs were exposed to 3 or 0.5 Gy of $\gamma$-irradiation. The culture medium was then replaced with fresh medium. After 7 days in culture, ovaries were fixed by incubation in Bouin's fixative for at least $1 \mathrm{~h}$, dehydrated in alcohol and embedded in paraffin for immunohistochemistry analysis. Some ovaries were cultured for $6 \mathrm{~h}$ after irradiation for the measurement of caspase activation.

\section{Quantification of follicle number}

We mounted $5 \mu \mathrm{m}$ sections on glass slides and stained them with haematoxylin-eosin. We counted the follicles on every fifth section, using the oocyte nucleus as a marker, and the stage of follicular development was determined as previously described (Guigon et al. 2003). Briefly, follicles were classified according to the shape and number of layers of somatic cells surrounding the oocyte: primordial (flattened cells), primary (one layer of cuboidal cells) and secondary (two partial or complete layers of cells). Intermediate follicles were defined as follicles containing mixed features of primordial and primary follicles.

\section{Immunohistochemistry}

Tissue sections were mounted on glass slides and boiled for $10 \mathrm{~min}$ in $10 \mathrm{mM}$ Tris (pH 10.6) to unmask antigens. Endogenous peroxidase activity was blocked by incubation with $3 \%$ hydrogen peroxide for $10 \mathrm{~min}$. Slides were incubated with the primary antibody overnight at $4{ }^{\circ} \mathrm{C}$. The primary antibodies used were mouse anti-pan-p63 (1:100; 4A4, Santa Cruz Biotechnology, Santa Cruz, CA, USA), mouse anti-p63 $\alpha$ (1:100; Santa Cruz Biotechnology), goat anti-HSP90 $\alpha / \beta$ (1:500; Santa Cruz Biotechnology), rabbit anti-cleaved caspase-3 (1:100; Asp 175, Cell Signaling Technology, Beverly, MA, USA) and rabbit anti-cleaved caspase-9 (1:100; Asp 353, Cell Signaling Technology) antibodies. Slides were washed in PBS and incubated for $30 \mathrm{~min}$ at room temperature with the corresponding biotinylated secondary antibody diluted 1:200 (Vector Laboratories, Peterborough, UK), and then for $30 \mathrm{~min}$ with the avidin-biotinperoxidase complex (Vector Laboratories). Peroxidase activity was visualised using 3,3'-diaminobenzidine (DAB) as the substrate. Finally, slides were counterstained with haematoxylin. We used similar protocols for anti-p53 (1:500, CM5, Novocastra, Newcastle, UK) and anti-p73 (1:100, H79, Santa Cruz Biotechnology) antibodies, except that $10 \mathrm{mM}$ citrate buffer ( $\mathrm{pH}$ 6.0) was used as the unmasking solution.

\section{Immunofluorescence}

Fixed ovary and testis sections were incubated for $1 \mathrm{~h}$ at room temperature with primary antibodies. The primary antibodies used were rabbit anti- $\gamma \mathrm{H} 2 \mathrm{AX}$ (1:200, Upstate - Cell Signaling Solutions) and anti-pan-p63 antibody (4A4). Sections were then washed in PBS and incubated for 45 min with donkey antimouse IgG-FITC (1:200) and goat anti-rabbit IgG-Cy3 antibodies(1:800, Jackson ImmunoResearch Laboratories, 
Newmarket, UK). Slides were mounted with Vectashield (Vector Laboratories) and analysed by conventional immunofluorescence microscopy using an Olympus Provis AX70 microscope.

\section{RNA extraction and semi-quantitative RT-PCR}

Total RNA was extracted from foetal and neonatal ovaries using the RNeasy Mini Kit (Qiagen), and 500 ng total RNA was reverse transcribed using the Omniscript RT kit (Qiagen) according to the manufacturer's instructions.

We investigated p63 expression by PCR amplification, as previously described (Petre-Lazar et al. 2007). All primers were obtained from Invitrogen (Invitrogen SARL). We used eight different primers to amplify all the various p63 isoforms in separate reactions (Fig. 4). We used $\beta$-actin as an internal control for RNA extraction and cDNA synthesis. The primers for $\beta$-actin were: $F 5^{\prime}$ AAGAGAGGTATCCTGACCCTG3' and R 5'GGCCATCTCCTGCTCGAAGT 3'.

Approximately 25-35 PCR cycles were performed with $1 \mu \mathrm{l}$ CDNA, 3 pmol of each primer, $0.2 \mathrm{mM}$ of each dNTP (Invitrogen), $2 \mathrm{mM} \mathrm{MgCl} 2$ (Invitrogen), $1 \cup$ Taq DNA polymerase (Invitrogen) in reaction buffer (20 mM Tris- $\mathrm{HCl}(\mathrm{pH} 8.4)$ and $50 \mathrm{mM} \mathrm{KCl}$ ) in a final volume of $25 \mu \mathrm{l}$. PCR was stopped before reaching the plateau. Amplification products were separated by electrophoresis in 1 or $2 \%$ agarose gels stained with ethidium bromide $(1 \mu \mathrm{g} / \mathrm{ml}$, Sigma). Each experiment was repeated at least thrice. No amplicon was obtained in reactions in which reverse transcriptase was omitted. All p63 PCR amplification products were sequenced by GENOME Express (Meylan, France).

\section{Protein extraction and Western blotting}

We resuspended $1 \mathrm{dpp}$ ovaries in homogenisation buffer (20 mM Tris base pH 8.0 (Sigma) containing $150 \mathrm{mM} \mathrm{NaCl}$ (Sigma), 0.5 mM EDTA (Sigma), 1\% Triton $\times 100$ (Sigma), 0.1\% SDS (Sigma), $10 \mathrm{mM}$ sodium fluoride (Sigma), $1 \mathrm{mM}$ sodium orthovanadate (Sigma), $10 \mathrm{mM} \beta$ glycerophosphate (Sigma) and protease inhibitor cocktail (1 tablet/10 ml extraction solution, Roche)). Samples were placed on ice for $30 \mathrm{~min}$, with homogenisation every $5 \mathrm{~min}$, and then centrifuged for $15 \mathrm{~min}$ at $4{ }^{\circ} \mathrm{C}$ and $11000 \mathrm{~g}$ to remove cellular debris. The protein concentration of the supernatant was then determined by the Bradford method. Finally, samples were frozen and stored at $-80{ }^{\circ} \mathrm{C}$ until Western blotting.

We subjected 30-100 $\mu$ g total protein to electrophoresis in an $8 \%$ polyacrylamide denaturing gel in Tris/glycine/SDS running buffer (25 mM Tris base (Sigma), $200 \mathrm{mM}$ glycine (pH 8.3; Sigma) and $0.1 \%$ SDS (Sigma)). The protein bands were blotted onto polyvinylidene difluoride membranes (Amersham Biosciences). Membranes were blocked by incubation for $1 \mathrm{~h}$ at room temperature in Tris-buffered saline (pH 7.4) containing 0.05\% Tween 20 (Sigma) and 5\% skimmed milk powder. They were then incubated overnight at $4{ }^{\circ} \mathrm{C}$ with the mouse anti-pan-p63 (4A4) or anti-p63 $\alpha$ antibodies diluted 1:1000 in the blocking solution. Membranes were incubated for $1 \mathrm{~h}$ at room temperature with a donkey anti-mouse IgG:HRP-linked antibody (Amersham) diluted 1:5000 in the blocking solution. Antibody-protein complexes were visualised with the ECL system (Amersham).

Membranes were stripped and reprobed for the mouse vasa homologue protein used as a reference. Briefly, the membranes were incubated for $15 \mathrm{~min}$ in a stripping buffer $(62.5 \mathrm{mM}$ Tris$\mathrm{HCl}$ (pH 6.8; Sigma), 2\% SDS (Sigma), $100 \mathrm{mM}$ $\beta$-mercaptoethanol (Sigma)). Vasa expression was detected as described above, using a mouse monoclonal anti-mvh/ddx4 (1:500) antibody (Abcam, Cambridge, UK). Actin protein was also used as a reference and was detected with a mouse monoclonal anti-actin antibody (CP01, Calbiochem Merck Eurolab).

\section{Data analysis}

Each data point represents the mean \pm S.E.M. of at least three independent experiments. Images show a representative experiment repeated at least thrice. Data were analysed using Student's $t$-test.

\section{Acknowledgements}

This work was supported by Electricité De France (EDF). We would like to thank $\mathrm{R}$ Frydman and the obstetrics and gynaecology staff of $A$. Béclère Hospital, who provided human tissue samples. We also thank $V$ Hanoux for valuable help with Western blot analysis, V Neuville and C Chauveau for taking care of the animals and J Sappa for critically reviewing this manuscript. $E$ T holds a fellowship from the CEA. M J G holds a fellowship from the Ministère de l'Education Nationale, de la Recherche et de la Technologie. The authors declare that there is no conflict of interest that would prejudice the impartiality of this scientific work.

\section{References}

Beumer TL, Roepers-Gajadien HL, Gademan IS, van Buul PP, Gil-Gomez G, Rutgers DH \& de Rooij DG 1998 The role of the tumor suppressor p53 in spermatogenesis. Cell Death and Differentiation 5 669-677.

Davison TS, Vagner C, Kaghad M, Ayed A, Caput D \& Arrowsmith CH 1999 p73 and p63 are homotetramers capable of weak heterotypic interactions with each other but not with p53. Journal of Biological Chemistry 274 18709-18714.

Gaiddon C, Lokshin M, Ahn J, Zhang T \& Prives C 2001 A subset of tumorderived mutant forms of p53 down-regulate p63 and p73 through a direct interaction with the p53 core domain. Molecular and Cellular Biology 21 1874-1887.

Gressner O, Schilling T, Lorenz K, Schulze Schleithoff E, Koch A, Schulze-Bergkamen H, Maria Lena A, Candi E, Terrinoni A, Valeria Catani $\mathbf{M}$ et al. 2005 TAp63alpha induces apoptosis by activating signaling via death receptors and mitochondria. EMBO Journal $\mathbf{2 4}$ $2458-2471$.

Guigon CJ, Mazaud S, Forest MG, Brailly-Tabard S, Coudouel N \& Magre S 2003 Unaltered development of the initial follicular waves and normal pubertal onset in female rats after neonatal deletion of the follicular reserve. Endocrinology 144 3651-3662.

Hamer G, Gademan IS, Kal HB \& de Rooij DG 2001 Role for c-Abl and p73 in the radiation response of male germ cells. Oncogene 20 4298-4304.

Hanoux V, Pairault C, Bakalska M, Habert R \& Livera G 2006 Caspase-2 involvement during ionizing radiation-induced oocyte death in the mouse ovary. Cell Death and Differentiation $\mathbf{1 4}$ $671-681$. 
Levrero M, De Laurenzi V, Costanzo A, Gong J, Wang JY \& Melino G 2000 The p53/p63/p73 family of transcription factors: overlapping and distinct functions. Journal of Cell Science 113 1661-1670.

Meirow D \& Nugent D 2001 The effects of radiotherapy and chemotherapy on female reproduction. Human Reproduction Update 7 535-543.

Melino G, De Laurenzi V \& Vousden KH 2002 p73: friend or foe in tumorigenesis. Nature Reviews. Cancer 2 605-615.

Mills AA, Zheng B, Wang XJ, Vogel H, Roop DR \& Bradley A 1999 p63 is a p53 homologue required for limb and epidermal morphogenesis. Nature $398708-713$.

Morita Y, Perez GI, Paris F, Miranda SR, Ehleiter D, Haimovitz-Friedman A Fuks Z, Xie Z, Reed JC, Schuchman EH et al. 2000 Oocyte apoptosis is suppressed by disruption of the acid sphingomyelinase gene or by sphingosine-1-phosphate therapy. Nature Medicine 6 1109-1114.

Petre-Lazar B, Livera G, Moreno SG, Trautmann E, Duquenne C, Hanoux V, Habert R \& Coffigny H 2007 The role of p63 in germ cell apoptosis in the developing testis. Journal of Cell Physiology 210 87-98.

Prise KM, Schettino G, Folkard M \& Held KD 2005 New insights on cell death from radiation exposure. Lancet Oncology 6 520-528.

Serber Z, Lai HC, Yang A, Ou HD, Sigal MS, Kelly AE, Darimont BD, Duijf PH, Van Bokhoven H, McKeon F et al. 2002 A C-terminal inhibitory domain controls the activity of p63 by an intramolecular mechanism. Molecular and Cellular Biology 22 8601-8611.
Skinner MK 2005 Regulation of primordial follicle assembly and development. Human Reproduction Update 11 461-471.

Suh EK, Yang A, Kettenbach A, Bamberger C, Michaelis AH, Zhu Z, Elvin JA, Bronson RT, Crum CP \& McKeon F 2006 p63 protects the female germ line during meiotic arrest. Nature 444 624-628.

Westfall MD \& Pietenpol JA 2004 p63: molecular complexity in development and cancer. Carcinogenesis 25 857-864.

Yang A, Kaghad M, Wang Y, Gillett E, Fleming MD, Dötsch V, Andrews NC, Caput D \& McKeon F 1998 p63, a p53 homolog at 3q27-29, encodes multiple products with transactivating, death-inducing, and dominantnegative activities. Molecular Cell 2 305-316.

Yang A, Schweitzer R, Sun D, Kaghad M, Walker N, Bronson RT, Tabin C, Sharpe A, Caput D, Crum C et al. 1999 p63 is essential for regenerative proliferation in limb, craniofacial and epithelial development. Nature $398714-718$.

Received 1 February 2007

First decision 27 February 2007

Revised manuscript received 16 August 2007

Accepted 3 October 2007 